Discussiones Mathematicae

\title{
WORM COLORINGS
}

\author{
Wayne Goddard $^{1}$, Kirsti Wash ${ }^{2}$ \\ AND \\ HONGHAI $\mathrm{XU}^{1}$ \\ ${ }^{1}$ Department of Mathematical Sciences \\ Clemson University, USA \\ ${ }^{2}$ Department of Mathematics \\ Trinity College CN, USA \\ e-mail: goddard@clemson.edu \\ kirsti.wash@trincoll.edu \\ honghax@clemson.edu
}

\begin{abstract}
Given a coloring of the vertices, we say subgraph $H$ is monochromatic if every vertex of $H$ is assigned the same color, and rainbow if no pair of vertices of $H$ are assigned the same color. Given a graph $G$ and a graph $F$, we define an F-WORM coloring of $G$ as a coloring of the vertices of $G$ without a rainbow or monochromatic subgraph $H$ isomorphic to $F$. We present some results on this concept especially as regards to the existence, complexity, and optimization within certain graph classes. The focus is on the case that $F$ is the path on three vertices.
\end{abstract}

Keywords: coloring, rainbow, monochromatic, forbidden, path.

2010 Mathematics Subject Classification: 05C15.

\section{REFERENCES}

[1] M. Axenovich and P. Iverson, Edge-colorings avoiding rainbow and monochromatic subgraphs, Discrete Math. 308 (2008) 4710-4723. doi:10.1016/j.disc.2007.08.092

[2] M. Axenovich, T. Jiang and A. Kündgen, Bipartite anti-Ramsey numbers of cycles, J. Graph Theory 47 (2004) 9-28.

doi:10.1002/jgt.20012 
[3] Cs. Bujtás, E. Sampathkumar, Zs. Tuza, C. Dominic and L. Pushpalatha, Vertex coloring without large polychromatic stars, Discrete Math. 312 (2012) 2102-2108. doi:10.1016/j.disc.2011.04.013

[4] Cs. Bujtás, E. Sampathkumar, Zs. Tuza, M.S. Subramanya and C. Dominic, 3consecutive C-colorings of graphs, Discuss. Math. Graph Theory 30 (2010) 393-405. doi:10.7151/dmgt.1502

[5] R. Cowen, Some connections between set theory and computer science, Lecture Notes in Comput. Sci. 713 (1993) 14-22. doi: $10.1007 / \mathrm{BFb} 0022548$

[6] W. Goddard, K. Wash and H. Xu, WORM colorings forbidding cycles or cliques, Congr. Numer. 219 (2014) 161-173.

[7] S.M. Hedetniemi, A. Proskurowski and M.M. Sysło, Interior graphs of maximal outerplane graphs, J. Combin. Theory Ser.B 38 (1985) 156-167. doi:10.1016/0095-8956(85)90081-4

[8] L. Lovász, On decomposition of graphs, Studia Sci. Math. Hungar. 1 (1966) 237-238.

[9] J.A. Telle and A. Proskurowski, Algorithms for vertex partitioning problems on partial k-trees, SIAM J. Discrete Math. 10 (1997) 529-550. doi:10.1137/S0895480194275825

[10] Zs. Tuza, Graph colorings with local constraints - a survey, Discuss. Math. Graph Theory 17 (1997) 161-228. doi:10.7151/dmgt.1049

Received 5 June 2014

Revised 17 November 2014

Accepted 17 November 2014 